\title{
Editorial
}

Digestive

Diseases

\section{Task-Shifting in Surveillance after Surgery for Colorectal Cancer: Addressing the Right Question?}

\author{
Kjetil Søreide ${ }^{a-c}$ \\ ${ }^{a}$ Clinical Surgery, Royal Infirmary of Edinburgh, University of Edinburgh, Edinburgh, UK; ${ }^{\text {b }}$ Department of \\ Gastrointestinal Surgery, Stavanger University Hospital, Stavanger, Norway; ${ }^{\circ}$ Department of Clinical Medicine, \\ University of Bergen, Bergen, Norway
}

Systematic follow-up after curative surgery for colorectal cancer continues to be a debated topic. After having gone through the shock of diagnosis, surgical treatment and possibly perioperative supportive oncological therapy, it is not surprising that most patients would prefer to be regularly monitored. Intuitively and emotionally it may be reassuring to be regularly seen and tested (e.g., by blood tests, imaging studies and clinical visits) for the possible early detection of recurrent disease. The practice of follow-up is widespread with several different protocols for systematic surveillance available. The evidence for doing this is however quite poor. Outcomes of several trials have demonstrated variable results at best, reflecting variation in type, tools and intensity of follow up [14]. The most recent Cochrane summary concluded that there is no survival benefit in those that are intensively followed compared to more lax surveillance [5], despite the fact that slightly more patients in the intense followup group are being offered curative surgery for a recurrence, should it occur.

Despite the data, systematic follow-up is done on a large scale across health care systems. The share volume of investigations and visits accumulates with the increasing number of patients treated with curative intent surgery. For obvious reasons, the volume is taking its toll on resources, including physicians' time and availability to continuously see patients. Notably, for stages I-III patients treated with

\section{KARGER}

(c) 2017 S. Karger AG, Basel

E-Mail karger@karger.com

www.karger.com/ddi curative surgery, $60-70 \%$ of these will be cured after surgery and never experience a clinically relevant recurrence. Thus, surveillance is for the select few patients who (a) are at risk for developing recurrence, (b) have a recurrence that is detected at an asymptomatic stage during surveillance and (c) who are eligible for second curative-attempt surgery. This group is arguably small and thus the current 'one size fits all' is consuming a large part of available health care resources in any health care system, relative to the benefit. Indeed, surveillance may not even be cost-effective based on data from the most recent FACS trial data [6]. In that trial, less than $17 \%$ of patients had a recurrence during follow up and among those who had, only a minority had surgery with curative intent. Thus, the question to whether resources are well spent for the few who actually have asymptomatic recurrence and can be treated with curative intent is still worth asking. A further notion in real-life practice is the lack of compliance to scheduled tests during surveillance. Due to the labour-intensive profile of surveillance, patients' compliance to scheduled intervals, the type of tests (e.g., lab test or endoscopy) and imaging studies (ultrasonography, CT or MRI) may decline over time, which may further decrease the intended value of the follow-up program. Indeed, previous studies have shown variable compliance to different tests $[7,8]$ and that clinicians tend to deviate from agreed national programmes if other available options are considered [9]. In the latter case, 
the most frequent change in surveillance was shifting some groups of patients previously seen by hospital physicians (e.g., surgeons) to be followed up by their general practitioner. This sort of task-shifting obligation from specialists to general practitioners is in some places seeing a further shift towards non-physician clinicians (NPCs).

Indeed, in the current issue of the Journal, the concept of task-shifting was evaluated by having NPCs performing the follow-up of patients after surgery for CRC [10] compared to those who saw a gastroenterologist or gastrointestinal surgeon. The research design was an observational study based on a population from a 7-hospital catchment region in the Rotterdam area in the Netherlands. In the study, patients recruited in the study were operated for colon cancer, so no patients with rectal cancer where studied in the current setting. Notably, treatment of colon and rectal cancer is different in many ways and they have different complication profiles both in the short- and long-term course. Thus, patients treated for rectal cancers are typically seen more frequently by their surgeons during the follow-up.

The Dutch programme described in the system follows a surgeon-led surveillance with or without the support of NPC for follow-up. In the current cohort, about $80 \%$ of patients were subject to an "intense" follow-up, associated with being seen by an NCP for surveillance. Baseline characteristics differed, for example, with more patients having stage III disease in the less-intense follow-up. For the entire cohort, there was no difference between intense and regular follow-up groups in overall survival. Stratified for other factors, such as center of care and NPC contact, there seemed to be a survival benefit in the intensely followed-up group, associated with NCP contact. One should bear in mind that a retrospective observational study may find associations that are not causally related. Further, the small group of regular follow-up patients, of which a larger number had stage III disease in addition to other unfavourable features, is likely to influence the resultant differences in survival. Thus, the conclusion that NCP-led surveillance is the immediate explanation for better survival needs to be checked against the background of the research design and potential confounding factors. Further, one would like to further dissect what the incentives and mechanisms are behind the apparently better compliance to surveillance in patients who have NCP-led surveillance. Is it simply reflecting a poor job done on the specialists' side in assuring that appropriate intervals and controls are scheduled? Or, does it reflect differences in patients' perception of disease and risk and, thus, willingness to undergo surveillance? Would other alternatives to surveillance control, such as patient-led booklets [11], that keep track of programme schedules be similarly effective (and less costly)? And, finally, but equally important in a cost containment era: how will knowledge of genetic profiles help us tailor the best surveillance (or even none) for those at risk?

With current knowledge of a limited survival benefit to the intense surveillance programmes, scrutinizing the role of surveillance to patients with colorectal cancer is a must. Asking the right question is a prerequisite in order to arrive at useful answers. The "why", the "who", "how" and "when" still appear to be followed by a definite question mark in this setting.

\section{References}

$>1$ Verberne CJ, Zhan Z, van den Heuvel ER, Oppers F, de Jong AM, Grossmann I, et al: Survival analysis of the CEAwatch multicentre clustered randomized trial. Br J Surg 2017; 104:1069-1077.

>2 Rosati G, Ambrosini G, Barni S, Andreoni B, Corradini G, Luchena G, et al: A randomized trial of intensive versus minimal surveillance of patients with resected Dukes B2-C colorectal carcinoma. Ann Oncol 2016;27:274-280.

-3 Primrose JN, Perera R, Gray A, Rose P, Fuller A, Corkhill A, et al: Effect of 3 to 5 years of scheduled CEA and CT follow-up to detect recurrence of colorectal cancer: the FACS randomized clinical trial. JAMA 2014;311:263-270.

4 Verberne CJ, Zhan Z, van den Heuvel E, Grossmann I, Doornbos PM, Havenga K, et al: Intensified follow-up in colorectal cancer patients using frequent Carcino-Embryonic Antigen (CEA) measurements and CEA-trig- gered imaging: results of the randomized "CEAwatch" trial. Eur J Surg Oncol 2015;41: 1188-1196.

5 Jeffery M, Hickey BE, Hider PN, See AM: Follow-up strategies for patients treated for nonmetastatic colorectal cancer. Cochrane Database Syst Rev 2016;11:CD002200.

-6 Mant D, Gray A, Pugh S, Campbell H, George $\mathrm{S}$, Fuller A, et al: A randomised controlled trial to assess the cost-effectiveness of intensive versus no scheduled follow-up in patients who have undergone resection for colorectal cancer with curative intent. Health Technol Assess 2017;21:1-86.

7 Körner H, Söreide K, Stokkeland PJ, Söreide JA: Systematic follow-up after curative surgery for colorectal cancer in Norway: a population-based audit of effectiveness, costs, and compliance. J Gastrointest Surg 2005;9:320 328.
8 Carey M, Sanson-Fisher R, Macrae F, Cameron E, Hill D, D'Este C, et al: Improving adherence to colorectal cancer surveillance guidelines: results of a randomised controlled trial. BMC Cancer 2017;17:106.

-9 Søreide K, Træland JH, Stokkeland PJ, Glomsaker T, Søreide JA, Kørner H: Adherence to national guidelines for surveillance after curative resection of nonmetastatic colon and rectum cancer: a survey among Norwegian gastrointestinal surgeons. Colorectal Dis 2012;14:320-324.

10 Coebergh van den Braak R, Lalmahomed Z, Hansen B, Büttner S, IJzermans J: Nonphysician clinicians in the follow up of resected patients with colorectal cancer. Dig Dis 2018;36: 17-25.

11 MacFater H, MacFater W, Hill A, Lill M: Individualised follow-up booklets improve recall and satisfaction for cancer patients. $\mathrm{N} \mathrm{Z}$ Med J 2017;130:39-45. 